\title{
Performance analysis through benchmarking and recognition of patterns
}

\author{
Univ.-Prof. Dr. Horst Wildemann \\ Department of Business Administration \\ with emphasis on Logistics \\ Technical University of Munich \\ Leopoldstrasse 145, 80804 Munich, Germany, \\ Tel: +49-89-36078-100 Fax: +49-89-36078-111
}

\begin{abstract}
Benchmarking should make it possible to expand the knowledge through methods and strategies and to derive appropriate actions for the own company. Central principal in benchmarking is the analogy-based comparison. An analysis within the framework of a study group with 23 companies shows that especially the search for reasonable data and the handling of the quantity of information makes many problems. Bilateral benchmarking projects show that regardless of the industrial branch learning effects can be realized through benchmark comparisons. Especially a comparison of business processes based on defined ratios and process-analysis is promising. As an approach to solving a problem for the identification of the best solution a recognition of patterns is being proposed. Recognition of patterns means the filtering of figures of different ratios and characteristics of best practices and efficient business processes out of a large data base. Not anymore single ratios, rather expressions in several dimensions are goals of benchmarking. On a basis of 282 european companies, patterns of organizational structures, cost structures, product attributes, and client relationships of successful companies are exemplarily being demonstrated.
\end{abstract}

Keywords:

Benchmarking, performance analysis, recognition of pattern, performance measurement, organizational learning, business process management, process benchmarking. 


\section{PROBLEM TASK}

Benchmarking means independent of markets, products and local point of views to aim for a comparison with the best practices. Benchmarking can be defined as a continuous and systematic process for the determination of exceptional methods and activities which allow a record performance. This record performance can be related to product development, process control, service supply or the use of certain methods. In benchmarking it depends on analyzing the efficiency of companies in different industrial branches and market segments. Effects of interdependencies between structural business features, used methods, rapideness in the implementation of objectives, and the fulfilment of factors of performance have to be made transparent to insure that target-orientated measures can be derived. Based on these requirements two different solutions attempts have been developed to be able to identify differences in positions: benchmarking of business processes and performance analysis through recognition of patterns.

\section{PROCESS-BENCHMARKING}

Within a framework of a study group containing 23 european industrial enterprises requirements of success of benchmarking were elaborated. As a result it showed that benchmarking of business processes is a particular successful approach. The general analysis of business processes gives the opportunity not only to analyze differences in the efficiency of processes, it also offers an evaluation of the effectiveness in terms of contribution to company success and in terms of fulfilment of client requests. An essential requirement of this is the guarantee of comparisons of business processes in-between companies of different industrial branches as well as the applicability of potential performance improvements. Therefore a framework was developed in co-operation with the companies which has been approved in several case studies (see figure 1).

In the scope of the purchasing for instance a comparison in-between different industrial branches has been performed. The branches were mechanical engineering and electrical engineering. The concentration was not that much on the comparison of processes as it was on methods which are suitable for identifying potentials in purchasing. It showed that there are several points of attachment in areas of purchasing organization, order processing, supplier selection and supplier development, which were being identified by the respective experts inside their own company as well as in foreign companies. The performance in the scope of purchasing measured by productivity-ratios such as volume of purchasing or supplier per employee was raised more than $15 \%$. 


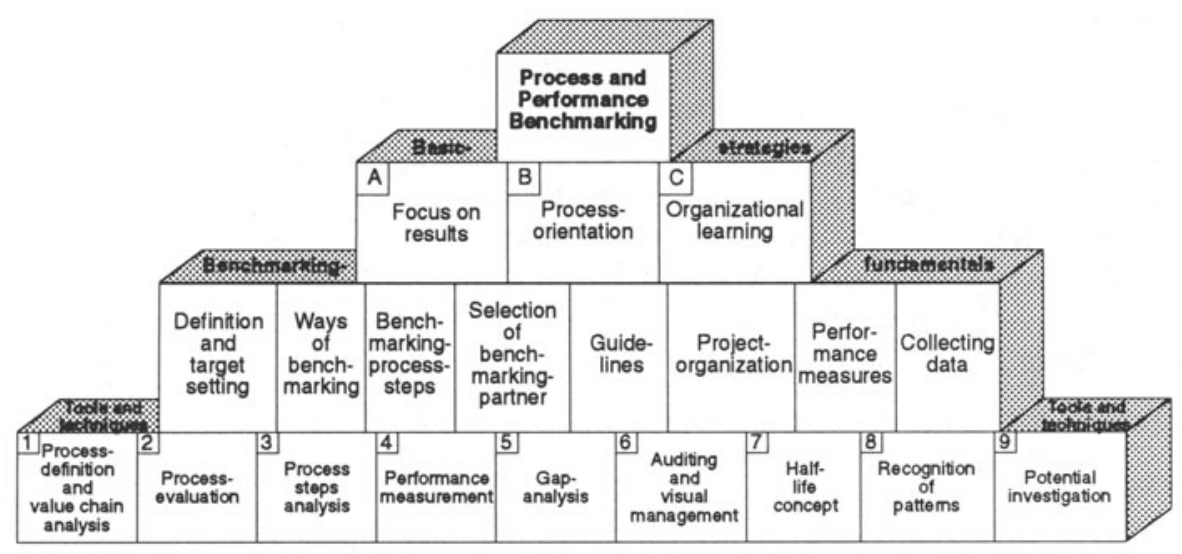

Figure 1 Benchmarking framework

Defining concrete performance-ratios at the beginning of the comparisons is of high importance to prove performance-gaps. In this context it is not necessary to analyze a multitude of isolated ratios rather than analyzing a few ratios which are distincted very differently in the companies. With this procedure differences of performance can be concluded with certainty. Besides a process analysis which is indispansible for the comprehension of the processes inside the compared companies, a procedure has been approved in which the respective best solution in the sense of a zerobased-budgeting can be combined to an ideal reference-model. The single solutions are being developed inside the involved companies or within the team. With this procedure of such a comparison with an ideal process for instance the lead time of a manufacturer of household utensils was reduced by more than $20 \%$.

\section{PERFORMANCE ANALYSIS THROUGH RECOGNITION OF PATTERNS}

In practice it shows that in the search of particularly efficient companies often the mistake of comparing a multitude of ratios is being done. The performance differentiate mostly only at certain points and the compared companies do not show in all analysed ratios significant advantages. In many cases comparative data exist only for certain parameters so that it is impossible to give clear interpretation. Due to the heterogeneity of the raw data and numerous influencing factors on the expressiveness of the gathered performance-ratios as well as the accelerated dynamic (declining validity of raw data), the practicability of a benchmarking process without electronic data-processing support cannot be done efficiently. A conventional data base system which exceptionally compares ratios supports exclusively the data management. However for the diversion of need for action and improvement potentials it gives too little support. 
Necessary therefore is a decision-support-system and an action-orientated diagnostic instrument. This was developed in strong co-operation with industrial companies within the study group. With the help of this system important parameters of successful industrial companies in form of descriptive attributes shall be identified. The evaluation of the performance of a company and its business processes is based on critical success dimensions such as logistics- and innovationperformance, or customer- and employee-orientation. Instead of a comparison of individual ratios in separate isolated levels of comparison a classification system was searched for, to offer a wider point of view for the evaluation of performance.

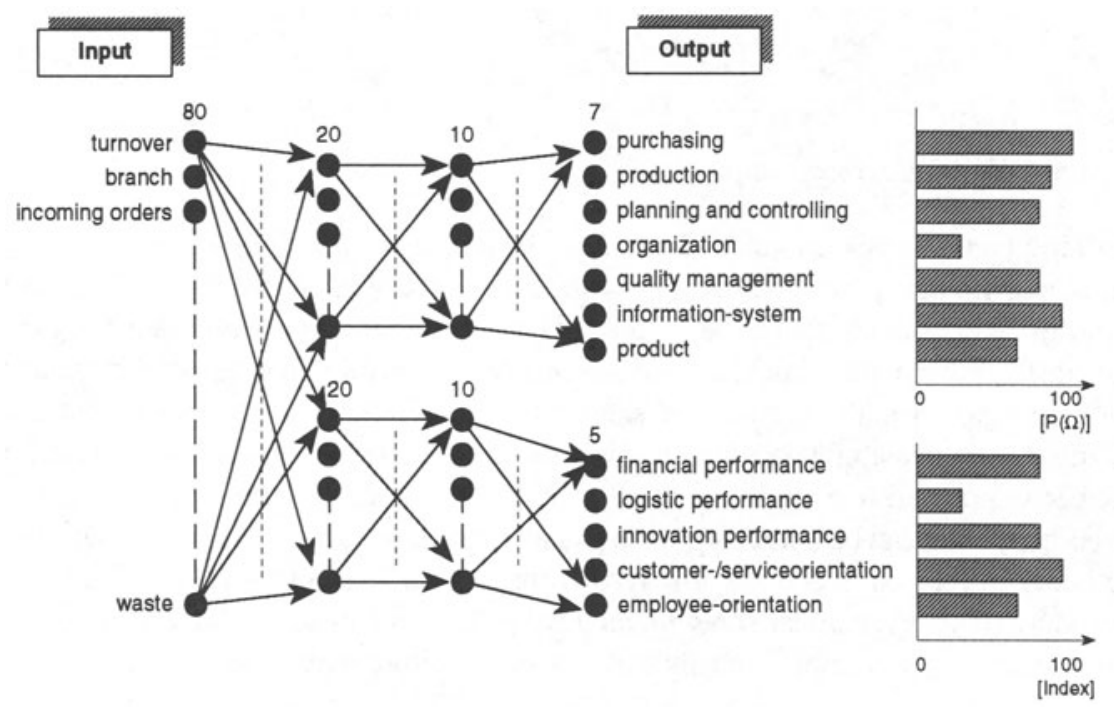

Figure 2 Classification through a neuronal network

Different kinds of classification methods, e.g. statistical methods like discriminance-analysis, geometrical methods and latest developments like neuronal networks have been analyzed and evaluated. What are neuronal networks? Artificial neuronal networks try to simulate the structure and the corresponding behaviour of a natural brain. They are able to improve their performance during the learning stage independently, which enables them to train complex patterns and to classify them with a very good reclassification-ratio. Statistical methods, e.g. usually assume independence within the data, what is not tenable in a business environment. The results of a geometrical classification are very dependent on the order of classification. After the evaluation of different possibilities, a neuronal network seemed to be the most efficient tool. Within the group of neuronal networks different designs have been considered, such as Hopfield-network, singleor multi-layer-perceptrons. Due to its high flexibility a multi-layer-perceptron was implemented. Figure 2 shows the structure the network is based on. The 
recognition of patterns has two levels: The company or a certain process is being pictured through a pattern and a classification to certain clusters can be done with this recognition-of-patterns-system.

After feeding the system with an initial pattern, the result of the classification are two new patterns, which give an idea about the probability of success for reorganizational measures in the predefined 7 areas and the company's performance in 5 dimensions (see figure 2).

The previous one-dimensional sight of a single criterion is being replaced by a pattern which can be characterized by an unlimited number of attributes. The objective therefore has to be trying to achieve the constellation of a target-pattern or the pattern of a successful company instead of the dimension of a single ratio. The decision over the performance of a certain pattern constellation can be made with a classification system. For the purpose of automation the classification-system was taught through learning samples. For successful manufacturing companies for instance it is possible to derive interdependencies in-between company size, vertical range of manufacture, and production equipment regardless of the industrial branch.

Large, successful companies are often characterised by a low vertical range of manufacture, and have efficient supplier so that employment fluctuation can be compensated through a transfer within the value chain. Successful, smaller companies though can even compensate sales volitality with a high vertical range of added value. This is possible if they can call a customer-orientated just-in-time production their own and if they are able to adjust their warehousing to their volume of output. Different pattern constellations exist for instance for the organization, production structure, cost structure, and for the sales structure for very successful and less successful companies even within an industrial branch (see figure 3).

\section{EXPECTED RESULTS}

With the benchmarking of business processes it is possible to identify potentials of improvement in costs, time and quality very fast. It is possible to realize organizational learning by identifying the respectively best solution and if the concrete implementation is realized within the own company. An essential advantage is that the learning process is not animated by theoretical or external knowledge, it is furthermore stimulated on a basis of a vivid comparison. 

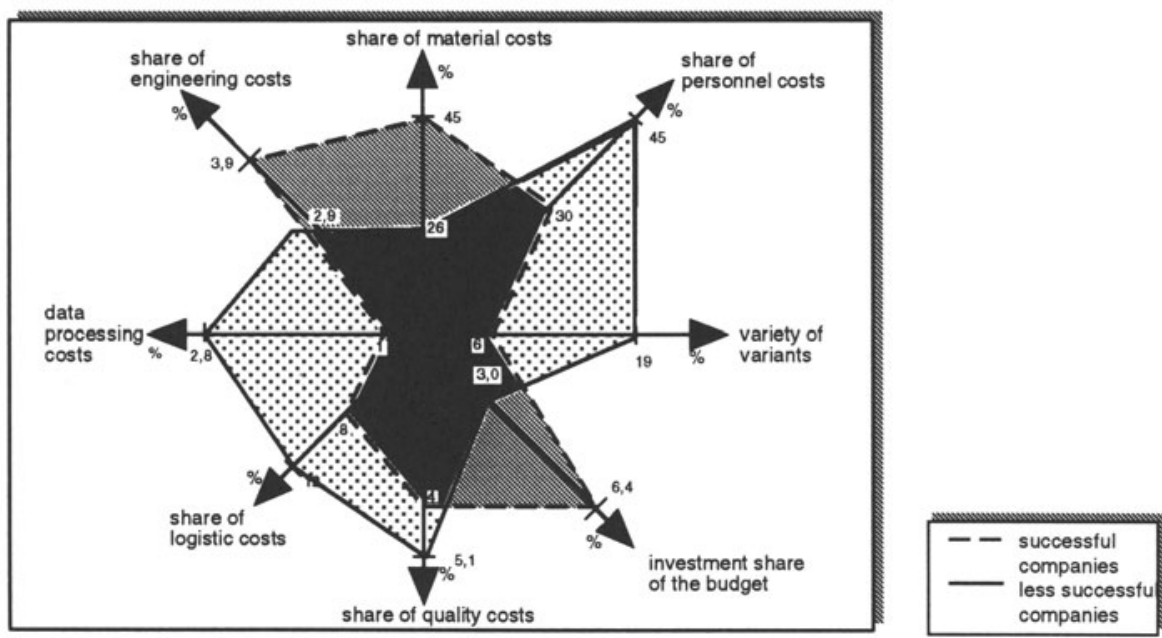

Figure 3 Cost structure (example)

The recognition of patterns enables the positioning of a company, the identification of several target patterns, as well as a purposive selection of potential promising reengineering priorities. It becomes possible to determine target ranges in distinctive dimensions and to raise the evidential value of benchmark comparisons. At the same time the costs for data collection decreases considerably. With the connection of company-related and process-related ratios it is possible to prognosticate potentials of improvement with a high accuracy. With the orientation of several ratios instead of focusing on just one best-practice-value the obtainability of goals can be raised which has a positive effect on the motivation.

\section{STAKEHOLDERS}

The results of the analysis are interesting for manufacturing concerns as well as for trade and service. The benefit of benchmarking is not attached to a certain company size or an industrial branch. Furthermore the composition of companies out of different industrial branches is the key for a successful benchmarking.

\section{STATE}

The named concepts have already been implemented in several case studies. Specific statements of achieved results in practice already exist. 


\section{REFERENCES}

Mertens, P. (1977): Die Theorie der Mustererkennung in den Wirtschaftswissenschaften, in: ZfbF, Heft 11, 29. Jg. 1977, S.777-794. Wildemann, H. (1995): Produktionscontrolling, 2. neub. Auflage, München 1995. Wildemann, H. (1996): Fertigungsstrategien, 3. Auflage, München 1996.

\section{BIOGRAPHY}

Prof. Dr. Horst Wildemann studied engineering (Dipl.-Ing.) and industrial management (Dipl.-Kfm.) in Aachen and Cologne. After several years of workingexperience as an engineer in the automobil-industry he graduated to Dr. rer. pol. in 1974. Stays abroad at the International Management Institute at Bruxelles and at american universities followed. In 1980 he completed his habilitation at the University of Cologne. Since 1980 he is working as a regular professor at the department of industrial management at the University of Bayreuth and Passau and the Technical University of Munich. He was offered a chair at the University of Stuttgart-Hohenheim and Dortmund, at the free University and Technical University Berlin and University of St. Gallen. Besides his research and lecture activities he is head of a research institute for strategic planning and logistics at the Technical University of Munich at which more than 50 people are employed. In 20 books and more than 200 essays, which all had been influenced by empirical findings, he showed new economic ways of how to organize and run a ,company with a future“. For leading industrial companies, Wildemann serves as a management consultant and as member of the supervisory board. 